\title{
GESTÃO URBANA E ARBORIZAÇÃO DAS CIDADES: DIAGNÓSTICO QUALITATIVO E QUANTITATIVO NO MUNICÍPIO DE SALGUEIRO, PERNAMBUCO, BRASIL
}

\author{
Felipe Luiz Lima de Paulo ${ }^{1}$
}

Ana Maria Siqueira Quirino ${ }^{2}$

\section{RESUMO}

As árvores são elementos de suma importância para a manutenção da qualidade de vida no meio urbano. A arborização urbana requer planejamento, pois existem muitas variáveis envolvidas, como o porte da cidade, o tipo de sistema viário, o modo como se estabelece a infraestrutura urbana e tambémo porte das espécies arbóreas. Diante disso, o presente trabalho tem por objetivo mostrar a relevância da arborização urbana, usando árvores nativas como forma de manutenção da biodiversidade regional em Salgueiro, Pernambuco. O estudo foi realizado no município de Salgueiro (Figura 01), localizado interior do Estado de Pernambuco, a cerca de $445 \mathrm{~km}$ da capital (Coordenadas 0804'27" S/ 3907'09" W). Para verificar os vegetais presentes na arborização urbana foi feita uma análise quantitativa. Encontraram-se 275 indivíduos arbóreos, pertencentes a seis famílias e seis espécies. As espécies mais comumente encontradas nas ruas foram: Ficus benjamina (72,7\%), Azadirachta indica (18,2\%), Archontophoenix Cunninghamii (3,6\%) e Prosopis juliflor (3,6\%). Citrus sinensis (1,8\%) e Melocactus bahiensis $(0,4 \%)$ são as espécies menos representativas. As espécies exóticas predominaram na arborização da cidade com cinco espécies representando $99,6 \%$ do total de indivíduos amostrados. Diante disso, considera-se indispensável o planejamento democrático da arborização urbana e a realização de um plano prevendo espécies.

Palavras-chave: planejamento e gestão urbana, arborização urbana, biodiversidade.

\section{MANAGEMENT AND URBAN STREET TREE OF CITIES: DIAGNOSIS AND QUALITATIVE QUANTITATIVE IN THE CITY OF SALGUEIRO, PERNAMBUCO, BRAZIL.}

\author{
ABSTRACT
}

\footnotetext{
1 Aluno especial da disciplina "Planejamento e Gestão Urbana" (MDU/UFPE). Mestre em Gestão do Desenvolvimento Local Sustentável (Universidade de Pernambuco). Professor do Curso de Pósgraduação em Educação Ambiental e Sustentabilidade para o Semiárido Pernambucano, Universidade Federal Rural de Pernambuco/Unidade Acadêmica de Serra Talhada (UFRPE/UAST). Fazenda Saco, s/n, Caixa Postal 063, Serra Talhada, PE, CEP 56900-000. E-mail: felipellpaulo@gmail.com

2 Aluna do Curso de Pós-graduação em Educação Ambiental e Sustentabilidade para o Semiárido Pernambucano, Universidade Federal Rural de Pernambuco/Unidade Acadêmica de Serra Talhada (UFRPE/UAST). Fazenda Saco, s/n, Caixa Postal 063, Serra Talhada, PE, CEP 56900-000. E-mail: anasiqueira17@hotmail.com
} 
Trees are very important elements for maintaining the quality of life in urban areas. The urban forestry requires planning because there are many variables involved, such as city size, the type of road system, how it establishes the urban infrastructure and also the size of the tree species. Therefore, this paper aims to show the relevance of urban vegetation using native trees as a way of maintaining regional biodiversity in Salgueiro, Pernambuco. The study was conducted in the municipality of Willow (Figure 01), located within the State of Pernambuco, about $445 \mathrm{~km}$ from the capital (coordinates 08 04 '27 "S / 39 。 $07^{\prime} 09^{\prime \prime} W$ ). To check the plants present in urban forestry a quantitative analysis was performed. We have found 275 tree species, belonging to six families and six species. The species most commonly found on the streets were: Ficus benjamina (72.7\%), Azadirachta indica (18.2\%), Archontophoenix cunninghamii (3.6\%) and Prosopis juliflor (3.6\%). Citrus sinensis (1.8\%) and melocactus bahiensis (0.4\%) are the least representative species. Exotic species predominated in greening the city with five species representing $99.6 \%$ of the sampled individuals. Therefore, it is essential to the planning ofurban greening and the realization of a plan providing species.

Keywords: planning and urban management, urban forestry, biodiversity.

\section{GESTIÓN Y URBANA DE LA CALLE DEL ÁRBOL DE CIUDADES: DIAGNÓSTICO Y CUANTITATIVA CUALITATIVO EN LA CIUDAD DE SALGUEIRO, PERNAMBUCO, BRASIL.}

\section{RESUMEN}

Los árboles son elementos críticos para mantener la calidad de vida en las zonas urbanas. Lasilvicultura urbana requiere una planificación, porque hay muchas variables que intervienen, como el tamaño de la ciudad, el tipo de sistema de carreteras, cómo se establece la infraestructura urbana y también el tamaño de las especies de árboles. Por lo tanto, este artículo tiene como objetivo mostrar la importancia de los árboles urbanos, el uso de árboles nativos como una forma de mantener labiodiversidad regional en Salgueiro, Pernambuco. El estudio se realizó en el municipio de Willow (Figura 01), situada en el Estado de Pernambuco, a unos 445 kilometros de la capital (coordenadas 08

- 04 '27 "N / 39 ०07'09" W). Para comprobar los vegetales presentes en la silvicultura urbana se realizó un análisis cuantitativo. Encontramos 275 árboles individuales pertenecientes a seis familias y seis especies. Las especies que se encuentran más comúnmente en las calles fueron: Ficus benjamina (72,7\%), Azadirachta indica (18,2\%), Archontophoenix cunninghamii (3,6\%) y Prosopis juliflor (3,6\%). Citrus sinensis $(1,8 \%)$ y bahiensis melocactus $(0,4 \%)$ son las especies menos representativas. Las especies exóticas predominaron en la ecologización de la ciudad con cinco especies que representan el 99,6\% de los individuos muestreados. Por lo tanto, es esencial para la planificación de las zonas verdes urbanas y la realización de un plan de previsión de las especies.

PALABRAS-CLAVE: la planificación y la gestión urbana, la silvicultura urbana, la biodiversidad.

\section{INTRODUÇÃO}

As árvores são elementos de suma importância "para a manutenção da qualidade de vida no meio urbano", tendo em vista que "funcionam como filtro 
ambiental, reduzindo a poluição atmosférica," amenizando "o calor, a insolação, a velocidade dos ventos e os ruídos", além disso, contribui no combate a erosão de solos desprotegidos, melhoria do ciclo hidrológico, redução das despesas com condicionamento térmico "e os frutos produzidos servem como fonte alimentar para os animais que as usam como abrigo" (MANUAL DE ARBORIZAÇÃO URBANA DE NATAL, 2009, p.3).

Historicamente, o que corrobora para a implantação da arborização em centros urbanos é o embelezamento que esta proporciona. Todavia, não se pode esquecer dos benefícios ecológicos, psíquicos e socioeconômicos que as árvores nos oferecem (SILVA, 2008).

A arborização urbana "não é uma tarefa simples, pois existem muitas variáveis envolvidas, como o porte da cidade, o tipo de sistema viário, o modo como seestabelece a infraestrutura urbana e também o porte das espécies arbóreas" (SILVA, 2008, p.1). Nesse sentido, o aspecto fundamental da arborização urbana é a escolha das espécies a serem utilizadas, visa racionalizar a ocupação do solo, de forma que o vegetal a ser plantado, sofra o mínimo possível de conflitos com os elementos urbanos - calçadas, modelo de calçadas, porte dos vegetais, distanciamento entre as árvores, altura da rede elétrica (SILVA, 2008; MANUAL DE ARBORIZAÇÃO URBANA DE NATAL,2009).

No semiárido nordestino podemos destacar a grande quantidade de vegetação xerófila na Caatinga. Entretanto, um dos principais problemas enfrentados na região tem sido a degradação e destruição dos recursos naturais, causados, principalmente, pela ação antrópica (PÔRTO; CABRAL; TABARELLI, 2004).

O município estudado situa-se no semiárido nordestino. Faz parte de uma das regiões menos conhecida no que diz respeito aos seus recursos naturais, principalmente sobre biodiversidade e é uma região pouco atendida e protegida. Essa região também é marcada por problemas socioambientais, destacando-se o problema da extração da lenha (SECTMA, 2004; PÔRTO; CABRAL; TABARELLI, 2004).

Com base nestes aspectos, o presente trabalho tem por objetivo mostrar a relevância da arborização urbana, usando árvores nativas como forma de manutenção da biodiversidade regional em Salgueiro, Pernambuco. 
PLANEJAMENTO E GESTÃo URBANA E A ARBORIZAÇÃO MUNICIPAL

Planejar e gerir o espaço público urbano não é uma tarefa simples e mecanicista. Requer envolvimento de atores diversos da sociedade para que haja bem comum, ou que se aproxime a isto. A complexidade nas relações socioculturais e as relações de poder entre os atores sociais, fazem com que a dinâmica do uso e ocupação do solo perpasse um mero instrumento legislativo emanado pelo governo.

Na democracia representativa, a dinâmica do planejamento, ou o ato deprever o futuro diante dos diversos aspectos que o influencia, é ojeriza daqueles estudiosos mais conservadores, pois perpassa pelo pensamento neoliberalista da delegação de poderes próprios do Estado. Contudo, é inevitável entender a importância do ato de planejar e de operacionalizar aquilo que foi previsto por meio dogerenciamento dentro da democracia representativa, em contraposição do centralismo do Estado. Não obstante a isto, em contraposição, até os marxisistas mais fervorosos, como Lenin, entenderam que planejar é necessário, mesmo entendendo que se trata de "simples instrumentos da classe dominante" (SOUZA,2006).

Longe de defender os instrumentos de planejamento e gestão urbana como salvadores da sociedade, demonstrados pelos zoneamentos de áreas valorizadas e a exclusão cada vez mais da população menos favorecida para áreas periféricas, por exemplo, entende-se que o problema não está na instrumentalização dos mesmos, contudo na real democracia das relações de poderes de um governo. A própria governança das cidades, termo cada vez mais usado entre pesquisadores do planejamento e gestão urbanos, tem, em sua própria origem como objeto de estudo a relação de conflito que há entre o principal (governante) e os agentes (administradores), que muitas vezes tem objetivos distintos na condução do governo (FONTES FILHO, 2003).

No caso da arborização dos espaços públicos urbanos, o que se observa muitas vezes é a valorização das áreas mais favorecidas referindo-se ao termo socioeconômico, em contraposição às áreas periféricas. Observa-se, também, a descaracterização, muitas vezes da vegetação nativa de uma região, em detrimento do avanço imobiliário e dos anseios temporal da sociedade, que, muitas vezes, não são coincidentes com o tempo em que a natureza precisa para se recuperar ou para se expandir. Observa-se também a necessidade muitas vezes da sociedade de se criar proximidade de uma região mais favorecida socioeconomicamente com aquela 
emergente, ou seja, acaba surgindo, nestes casos, a proliferação de espécies que não são nativas de uma localidade ou município.

\section{MATERIAL E MÉTODOS}

\section{A. Área de estudo}

O estudo foi realizado no município de Salgueiro (Figura 01), localizado interior do Estado de Pernambuco, a cerca de $512 \mathrm{~km}$ da capital (Coordenadas 0804'27" S/ 3907'09" W), esta situado na mesorregião do Sertão de Pernambuco e na microrregião de Salgueiro, limitando-se ao Norte com Penaforte (CE) ao Sul com Belém de São Francisco e a Oeste com Terra Nova, o local esta com 420 metros de elevação. Sua extensão territorial é de aproximadamente $1.733,7 \mathrm{~km}^{2}$. De acordo com o senso do Instituto Brasileiro de Geografia e Estatística (2013), a cidade possui população estimada de 56.641 habitantes. Possui temperatura média anual de $26 \mathrm{C}^{\circ}$, clima tropical chuvoso e vegetação de Caatinga xeromórfica.

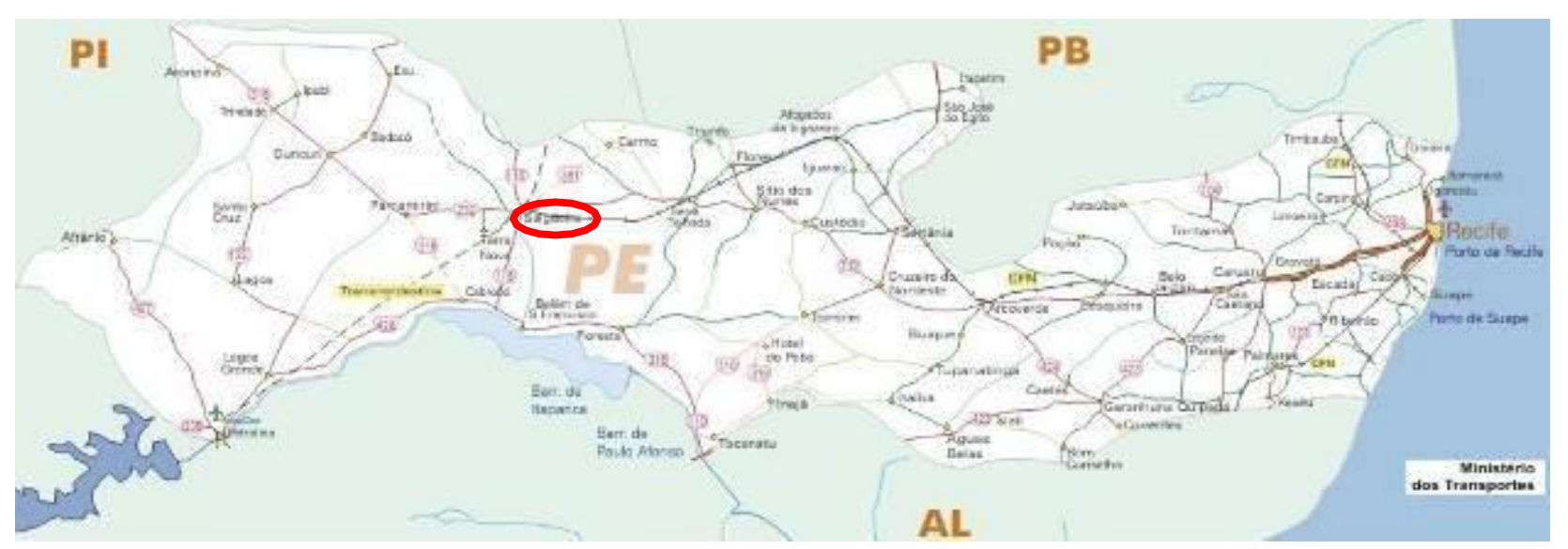

Figura 01: Mapa do Estado de Pernambuco, pontuando a região do Sertão do Pajeú, com destaque para o município de Salgueiro, Pernambuco, Brasil. Fonte: Ministério dos Transportes.

\section{B. Coleta de dados}

Os dados para a revisão de literatura foram coletados online, em revistas, periódicos e livros, através de pesquisas na internet, buscando pelas palavras chave: 
Volume 10, Número 4, 2014

Dilemas da

Sustentabilidade Urbana

arborização urbana; qualidade de vida; espécies nativas; legislação ambiental municipal de Salqueiro e Plano Diretor, no período de 28 de janeiro de 2014 a 15 de feverreiro de 2014. Posteriormente, foi feito um levantamento in loco de todos os indivíduos vegetais existentes nas praças do município e nas calçadas das vias públicas, através da observação direta e registro fotográfico. Para o estudo, foram consideradas tanto espécies nativas como também exóticas, de porte arbustivo a arbóreo. A identificação das espécies, enquadradas nas condições mencionadas, com seus respectivos nomes científicos e famílias, ocorreu através da consulta bibliográfica baseada em LORENZI (2008) e BACKES; IRGANG (2002).

\section{RESULTADOS E DISCUSSÃO}

Foram identificadas seis famílias, seis espécies e 275 indivíduos (Tabela 01). A família mais representativas foram Moraceae (200 indivíduos), seguida de Meliaceae (50 indivíduos), Palmae (10 indivíduos) e Leguminosae (10 indivíduos). As espécies mais comumente encontradas nas ruas são Ficus benjamina, Azadirachta indica, Archontophoenix Cunninghamii e Prosopis juliflor (Figura 02). Sendo Citrus sinensis (4) e Melocactus bahiensis (1) as espécies menos representativas.

Tabela 01: Litas das famílias e de espécies arbóreas encontradas na arborização das vias públicas do município de Salgueiro, PE. Fonte: dados da pesquisa.

\begin{tabular}{c|c|c|c|c} 
Família & Nome científico & Nome popular & $\begin{array}{c}\text { Nativa (N) ou } \\
\text { Exótica (E) }\end{array}$ & $\begin{array}{c}\text { Quantidades } \\
(\mathbf{n} \mathbf{0})\end{array}$ \\
\hline Rutaceae & Citrus sinensis & Laranjeira & $\mathrm{E}$ & 4 \\
\hline Palmae & $\begin{array}{c}\text { Archontophoenix } \\
\text { Cunninghamii }\end{array}$ & Palmeira & $\mathrm{E}$ & 10 \\
\hline Moraceae & Ficus benjamina & Fícus & $\mathrm{E}$ & 200 \\
\hline Cactaceae & Melocactus bahiensis & Coroa de frade & $\mathrm{N}$ & 1 \\
\hline$\underline{\text { Meliaceae }}$ & Azadirachta indica & Nim & $\mathrm{E}$ & 50 \\
\hline$\underline{\text { Leguminosae }}$ & Prosopis juliflor & Algaroba & $\mathrm{E}$ & 10 \\
\hline & & & & Total=275
\end{tabular}




\section{Dilemas da} Sustentabilidade Urbana
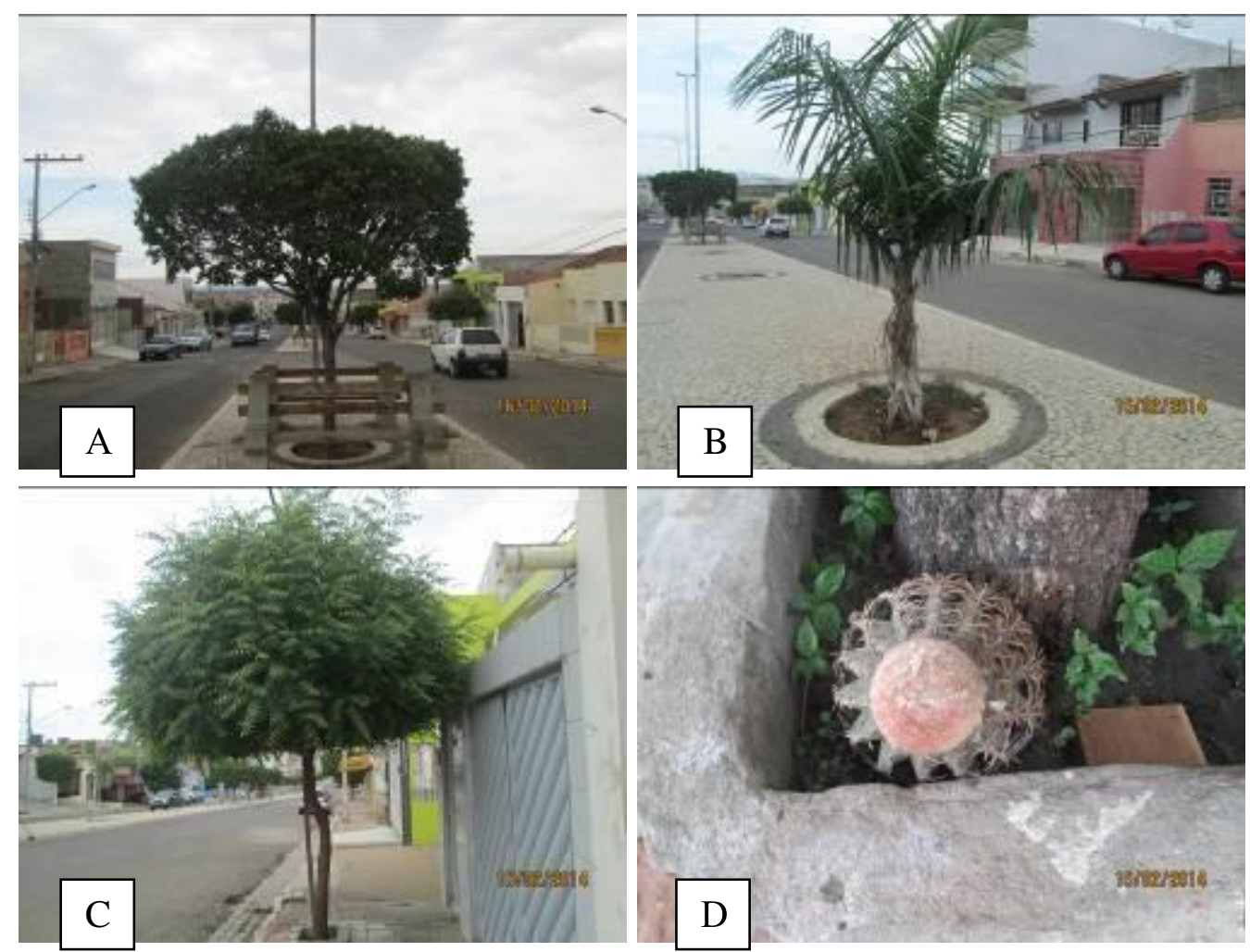

Figura 02: Espécies de plantas encontradas nas vias públicas de Salgueiro, PE. A- Ficus benjamina; B- Archontophoenix Cunninghamii; C- Azadirachta indica; D- Melocactus bahiensis. FONTE: Os autores, 2014.

Em número de espécies, as plantas exóticas (Figura 03) são mais predominantes na arborização da cidade com 274 indivíduos, estando representadas em $99,6 \%$ do total de indivíduos amostrados. 


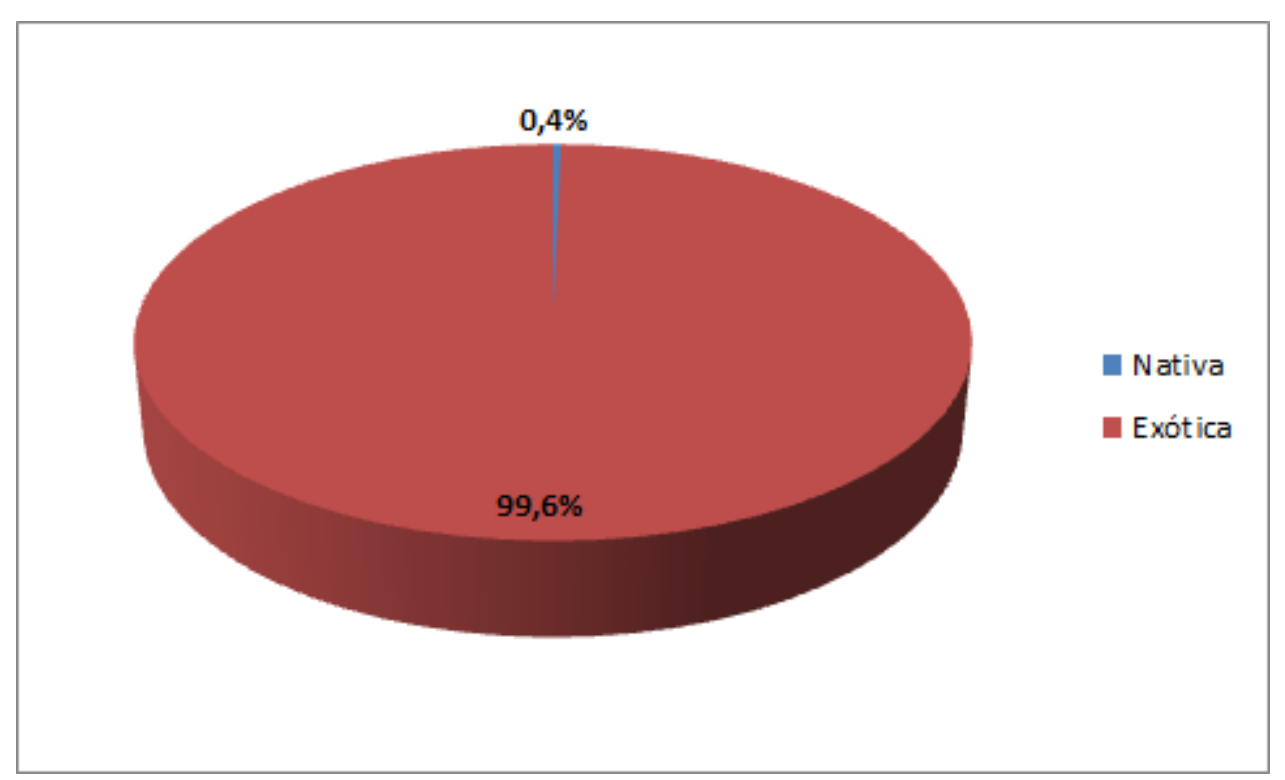

Figura 03: Porcentagem de indivíduos nativos e exóticos ocorrentes na arborização urbana de Salgueiro, PE. Fonte: dados da pesquisa.

Dentre as famílias amostradas (Figura 04) a que apresentou maior diversidade foi a Moracea, seguida da Rutaceae, sendo todas exóticas.

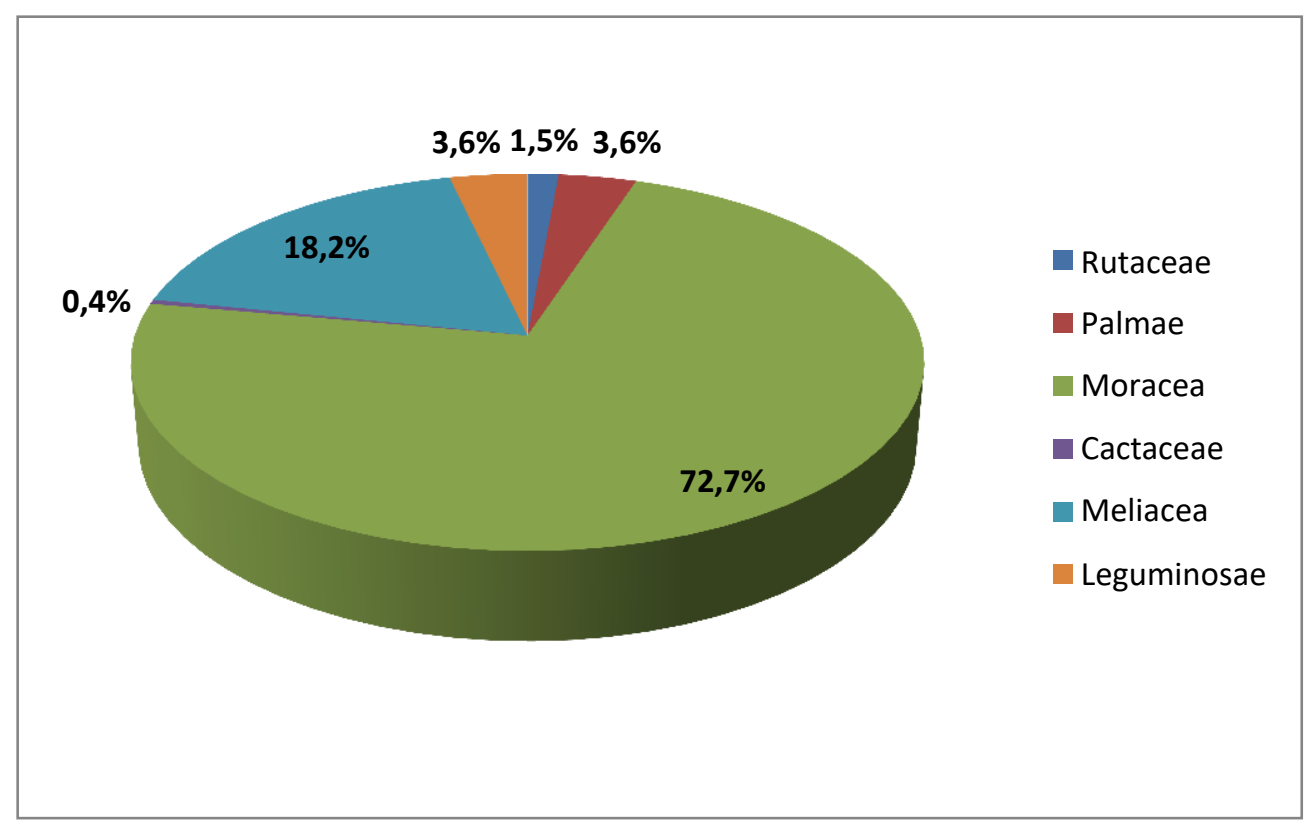

Figura 04: Principais famílias existentes na arborização urbana de Salgueiro, PE.

Fonte: dados da pesquisa 
"Dentre as situações inadequadas e presentes nos conflitos da arborização urbana" (Melo, et al, 2007, p.14) no município de Salgueiro, PE, pode-se destacar o avanço da copa para a rua, o avanço da copa para a casa, obstrução de passagem de pedestres nascalçadas, poda feita de maneira inadequada e problemas ocasionados pela raiz. O mesmo resultado foi encontrado por Melo et al. (2007) em diagnóstico qualitativo e quantitativo da arborização urbana realizado no Paraíba. Com relação ao contato das árvores amostradas com a rede elétrica, 215 indivíduos não apresentam problemas com fiação e 60 indivíduos apresentam problemas relacionados com a fiação da rede elétrica e telefônica.
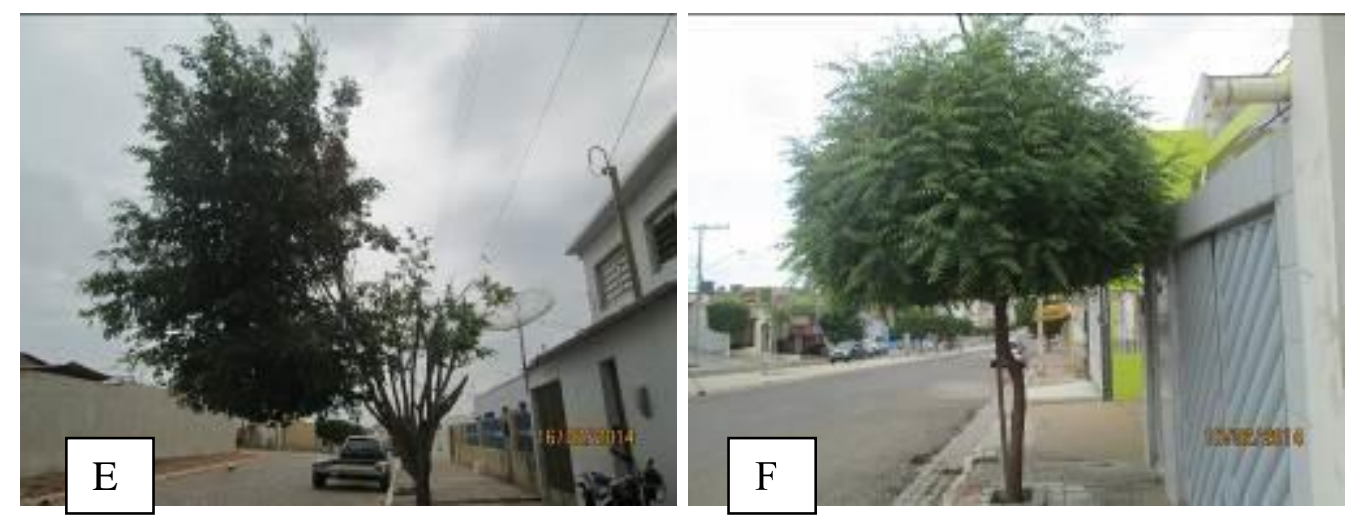

Figura 05: Problemas relacionados há ausência de planejamento relacionado à arborização urbana de Salgueiro, PE. E- Poda feita de maneira inadequada para a copa da árvore não atingir a rede elétrica; FNim obstruindo a passagem na calçada. FONTE: Os autores, 2014.

A escassez de árvores em vias públicas gera consequências para a biodiversidade, o que pode afetar também a qualidade de vida da população local.

\section{CONSIDERAÇÕES FINAIS}

A análise dos resultados obtidos mostra que das 275 espécies inventariadas, a mais frequente foi o fícus (Ficus benjamina). O município de Salgueiro, PE possui uma arborização urbana com baixa diversidade. Isto pode ser reflexo do rápido crescimento de árvores que acaba proporcionando sombra para a população. 
Entretanto, isso pode afetar a biodiversidade da localidade e descaracterizar a sua paisagem natural.

Corroborando os achados de Raber \& Rebelato (2010, p.14), "em número de espécies, as plantas exóticas são mais predominantes na arborização da cidade". O que se faz necessário a:

\footnotetext{
"troca oportuna de exemplares exóticos por nativos uma vez que resultaria no aumento da diversidade arbórea do município, pois uma maior diversidade garante uma maior proteção dos exemplaresao ataque de pragas e doenças, contribui para a manutenção da flora local e contribuipara a conservação das espécies e da biodiversidade." (Raber \& Rebelato, 2010, p.14)
}

Considera-se, assim, indispensável o planejamento da arborização urbana de forma democrática, envolvendo centros de pesquisas e sociedade civil em geral, por exemplo, e a realização de um plano, prevendo critérios e técnicas adequadas de plantios e manutenções, para evitar conflitos com os elementos urbanos.

A arborização de Salgueiro, PE apresenta diversos problemas relacionados à escolha das espécies utilizadas, como por exemplo, a copa das árvores que esta atingindo a rede elétrica, alguns exemplares arbóreos estão danificando calçadas, necessitando de reparos e a obstrução da passagem dos pedestres, fato comum para diversas cidades brasileiras.

Para este problema, recomenda-se a elaboração de uma listagem de espécies sugeridas para plantio, para orientar a comunidade em geral e a implementação de um plano municipal de arborização urbana acompanhando e controlando de maneira efetiva por algum órgão do meio ambiente (elaborado de forma coletiva).

\section{REFERÊNCIAS}

BRASIL. Prefeitura Municipal de Salgueiro: Lei ํํ. 1.635/2008. 2013.

BACKES, P.; IRGANG, B. Árvores do Sul: Guia de Identificação \& Interesse Ecológico. Santa Cruz do Sul: Instituto Souza Cruz, 2002. 
FONTES FILHO, Joaquim Rubens. Governança organizacional aplicada ao setor público. In.: VIII Congresso Internacional del CLAD sobre Reforma del Estado y de la Administración Pública. Paraná, 28-31 out., 2003 (digital).

IBGE. Salgueiro. Disponível em:

<http://cidades.ibge.gov.br/xtras/temas.php?codmun=261220\&idtema=119\&search=pernambuco\%7Csal gueiro\%7Cestimativa-da-populacao- 2013>. Acesso em: 10. fev. 2014.

LORENZI, H. Árvores Brasileiras: Manual de identificação e cultivo de plantas arbóreas do Brasil, Vol. 2. 3. ed. Nova Odessa: Instituto Plantarum, 2008.

MANUAL DE ARBORIZAÇÃO URBANA DE NATAL. Secretaria Municipal de Meio Ambiente e Urbanismo. SEMURB, Natal; 2009.

MELO, R. R.; LIRA FILHO, J. A.; JÚNIOR, F. R. Diagnóstico qualitativo e quantitativo da arborização urbana no Bairro Bivar Olinto, Patos, PR. Revista da Sociedade Brasileira de Arborização Urbana. Piracicaba. v.2, n.1, p.64-80, 2007.

SILVA, L.; M. REFLEXÕES SOBRE A IDENTIDADE ARBÓREA DAS CIDADES. Rev. SBAU, Piracicaba, v.3, n.3, p. 65-71; 2008.

PEREIRA, et al. O uso de Espécies Vegetais, como Instrumento de Biodiversidade da Avifauna Silvestre, na Arborização Pública: o caso de Recife. Atualidades Ornitológicas. Olinda, n. 125, p. 115, maio/junho, 2005.

PÔRTO, K. C.; CABRAL, J. J. P.; TABARELLI, M; (Org.). Brejos de altitude em Pernambuco e Paraíba: história natural, ecologia e conservação. Brasília : Ministério do Meio Ambiente, 324p., 2004.

RABER, Aline Pazinato; REBELATO, Gisele Sana Rebelato. ARBORIZAÇÃO VIÁRIA DO MUNICÍPIO DE COLORADO, RS - BRASIL: ANÁLISE QUALI-QUANTITẢTIVA. REVSBAU, Piracicaba - SP, v.5, n.1, p.183-199, 2010

SECTMA-CONSELHO NACIONAL DA RESERVA DA BIOSFERA DA CAATINGA. 2004. Cenários para o Bioma Caatinga. Secretaria de Ciência, Tecnologia e Meio Ambiente. Recife, 283p, 2004.

SOUZA, Marcelo Lopes. A PRISÃO E A ÁGORA: reflexões em torno da democratização do planejamento e da gestão das cidades. Rio de Janeiro: Bertrand, 2006. 